\title{
Clinical features of a series of non-surgical patients with focal cortical dysplasia and epilepsy
}

\author{
Gustavo Seifer, Juan Pablo Princich, Silvia Kochen
}

Center for Clinical and Experimental Neuroscience, Epilepsy, Cognition and Behavior, IBCN, School of Medicine, Epilepsy Section, Division of Neurology, Ramos Mejía Hospital, UBA, CONICET. Buenos Aires, Argentina

\section{Email address:}

seifergustavo@yahoo.com.ar (G. Seifer)

\section{To cite this article:}

Gustavo Seifer, Juan Pablo Princich, Silvia Kochen. Clinical Features of a Series of Non-Surgical Patients with Focal Cortical Dysplasia and Epilepsy. American Journal of Psychiatry and Neuroscience. Vol. 2, No. 1, 2014, pp. 8-12. doi: 10.11648/j.ajpn.20140201.12

\begin{abstract}
Most published series of patients with focal cortical dysplasia and epilepsy are surgical or pathological series of pediatric cases. Patients have a high frequency of seizure. The description of clinical features and the frequency patterns of non-surgical adult patients are less describe. We retrospectively reviewed the clinical records of adult patients ( $>18$ years of age) that visited the Epilepsy Centre of the Ramos Mejía Hospital in the city of Buenos Aires between 2010 and 2012 . We included all cases with confirmed diagnosis of epilepsy and MRI diagnosis of focal cortical dysplasia by standardized $1.5 \mathrm{~T}$ MRI. We analyzed the following variables: sex, age at seizure onset, seizure types, seizure frequency, presence of abnormal neurological exam, family history of epilepsy, existence of perinatal insults and electroencephalography or video-EEG with at least one ictal recording. We included 20 patients since 2010-2012. Mean age of our population was 25.9 years (9-46 years), 7 females, 13 males, they all had a negative family history of epilepsy, and only two patients had pathological neurological exam, both with mild contralateral paresis. Mean seizure onset age was 5.71 years (2 months-17 years) and the average frequency was 5.1 seizures per month (0-15). Two patients became seizure-free after adjusting antiepileptic drugs. Focal seizures were presented in the $100 \%$ of our population. The low frequency of seizure emphasizes the heterogeneity of these patients and the importance of the correct use of antiepileptic drugs schemes, as well as it can be dynamic over time. A proportion of medically resistant patients with cortical dysplasia are poor surgical candidates because the lesion cannot be completely identify or removed if it involves eloquent areas of the cortex. With the development of new drugs and the correct choice of treatment schedules is expected that more patients with focal cortical dysplasia would be treated successfully.
\end{abstract}

Keywords: Epilepsy, Focal Cortical Dysplasia, Malformations of Cortical Development

\section{Introduction}

Malformations of cortical development (MCD) are a heterogeneous group of focal and diffuse cortical derangements whose pathological features depend largely on the timing of the defect in the developmental process and to a lesser extent on its cause $[1,2]$. MCD are considered the second most common cause of medically resistant focal epilepsy in adults after hippocampal sclerosis [3]. In patients with resistant epilepsy, the MCD have been observed in $8-12 \%$ of cases [4] and in up to $14 \%$ of children with resistant epilepsy and mental retardation $[5,6]$.

Focal cortical dysplasia (FCD) is the most frequent type of MCD. They were first described by Taylor et al. in 1971 [7] in resected tissue from epileptic patients as focal cortical lesions with severe cortical dyslamination, the presence of dysmorphic and inmature neurons throughout all but the first layer of the cortex, and, in most cases, cells of uncertain origin called "balloon cells" (BCs). FCDs are highly epileptogenic lesions frequently associated with a wide spectrum of clinical manifestations that include epilepsy, mental retardation and neurological deficits.

With the improvements of genetics and the introduction of modern neuroimaging since the late 1980s, the detection of FCD in patients with resistant epilepsy has increased markedly. With better detection it is also becoming clear that FCD constitutes a wide spectrum of histopathologic and clinical presentations much broader than initially suspected [2]. This ranges from mild cortical dysplasia (currently termed FCD type I) presenting with difficult visualization by MRI, to more severe and obvious by MRI (FCD type II or III) presenting mostly in young children with aggressive 
seizures and status epilepticus.

Most published series of FCD are surgical series of patients with drug resistant epilepsy and a very high seizure frequency even daily. The aim of our work is to contribute to a better characterization of patients with epilepsy associated with FCD visible on MRI without the bias of being a surgical series. We emphasize the heterogeneity of this group of patients and that seizure frequency is not always as high as previously published by other authors.

\section{Material and Methods}

We retrospectively reviewed the clinical records of adult patients ( $>18$ years of age) that visited the Epilepsy Centre of the Ramos Mejía Hospital in the city of Buenos Aires, between 2010 and 2012. We included all cases with confirmed diagnosis of epilepsy and whose MRI had identified FCD. A standardized 1.5T MRI that included T1and T2-weighted, inversion recovery and fluid-attenuated inversion recovery acquisitions was used. We only included cases in which the opinion of two different neurologists (one of them specialized in imaging techniques) agreed on the diagnosis.

We analyzed the following variables: sex, age, age at seizure onset, seizure types, seizure frequency, presence of abnormal neurological exam, mental retardation, family history of epilepsy or neurological disease (first degree relatives), the existence or prenatal (intrauterine) or perinatal insults, electroencephalography (EEG) or video-EEG (VEEG) with at least one ictal recording. A patient was considered mentally retarded when his IQ was below 70 according to Wechsler Adult Intelligence Scale (WAIS test) [8]. Seizures were classified as focal or generalized based on semiology according to the reports of the International League Against Epilepsy (ILAE) Commission on Classification and Terminology of 1981, 1989 and the last report published in 2010 [9]. Cases that had missing clinical or epidemiological data were excluded from the study.

The MRI scans were retrospectively and independently reviewed by means of conventional visual analysis by two experienced neuroradiologists (JP and DC) who were involved in our epilepsy protocol. Discrepancies between the reviewers led to a combined re-evaluation of the case, if consensus was not achieved, the patient was excluded from the study. The following individual MRI aspects were considered: (1) cortical thickening; (2) GM/WM junction blurring; (3) WM hypersignal on TSE-T2WI and FLAIR-T2WI; (4) WM hyposignal on T1WI; (5) tapering of WM signal changes towards the ventricle (the transmantle sign); (6) cortex hypersignal on T2WI; and (7) gyral/sulcal anomalies. Subcortical WM hyperintensity on T2WI and hypointensity on T1WI were subjectively judged as absent or present. A specific MRI diagnosis of FCD was made when a combination of its distinctive signs was encountered [10-12]. When the MRI data suggested a dysplastic lesion but some particular features of FCD were missing, the lesion was discarded. Our series did not include any pediatric patient (neonates or infants), for whom different imaging variables should be considered [11].

The dysplastic lesions were classified on the basis of their anatomical location, they were also classified as lobar (involving a single lobe) or multilobar when they involved at least two lobes. They also were classified according to their extension using quantitative criteria; the small lesions included those located deeply along or at the bottom of sulci $[12,13]$.

To classify whether patients were responsive to treatment, we used the latest definition proposed by the ILAE. According to this definition, patients were considered to have drug resistant epilepsy when at least two adequate and tolerated antiepileptic drug (AED) schedules failed to achieve sustained seizure freedom [14]. Each AED was used at the highest tolerated dose and AED levels, available for the classic drugs, were measured for different purposes (to establish adherence, toxicity, etc.). In accordance with ILAE Treatment Guidelines, [15] carbamazepine is most often selected in our center as the first choice for adults with partial onset seizures. Monotherapy is always seen as the best alternative whenever possible, and newer drugs are usually used as add-on therapies.

\section{Results}

\subsection{Clinical Data}

We included 20 patients since 2010-2012. Mean age of our population was 25.9 years (9-46 years), 7 females, 13 males, they all had a negative family history of epilepsy, and only two patients had pathological neurological exam, both with mild contralateral paresis. Past medical history before epilepsy onset was relevant in 8 patients, five had febrile seizures $(25 \%)$, one case a perinatal infection $(5 \%)$, and two suffered of perinatal hypoxia/anoxia $(10 \%)$. Three patients had mild-to-severe mental retardation. Mean seizure onset age was 5.71 years ( 2 months -17 years) and the average frequency was 5.1 seizures per month $(0-15)$. Two patients became seizure-free after adjusting antiepileptic drugs. Focal seizures were presented in the $100 \%$ of our population. The initial semiology was as follows: 7 subjects had focal clonic seizures, 6 patients had focal asymmetrical tonic seizures, 2 patients had contralateral versive oculocephalic seizures, 3 patients had dyscognitive phenomena with impairment of memory, speech, attention, and perception, and one patient had auditory phenomena as initial manifestation and another subject had hyperkinetic pedal movements. All patients presented a single seizure type. Seven patients (35\%) had secondarily generalized seizures. In the two cases of seizure free, clinical semiology was assumed taking account that the story of the patient and family members committed to their care match. None of our patients had history of status epilepticus, epilepsy partialis continua, reflex seizures or history of infantile spams (Table 1). 
Table 1. Mean clinical features

\begin{tabular}{|c|c|c|c|c|c|c|}
\hline Patient & Age (years) & Onset sz (years) & Personal history & Sz/month & MRI (FCD) & Initial sz semiology \\
\hline 1 & 15 & 5 & No & $5 /$ month & $\begin{array}{l}\text { Left frontal - medial aspect of } \\
\text { superior gyrus }\end{array}$ & Tonic elevation $\mathrm{R}$ arm \\
\hline 2 & 32 & 4 & Prolonged labor & 4/month & $\begin{array}{l}\text { Left frontal - medial aspect of } \\
\text { superior gyrus }\end{array}$ & Tonic posture of $\mathrm{R}$ arm \\
\hline 3 & 26 & 2 & Febril seizure & 5/month & Left Parahippocampus & Auditory phenomena \\
\hline 4 & 30 & 10 & Febril seizure & 6/month & $\begin{array}{l}\text { Right temporal - anterior } \\
\text { middle gyrus }\end{array}$ & $\begin{array}{l}\text { mnemonic (deja vu) and dyscognitive } \\
\text { phenomena }\end{array}$ \\
\hline 5 & 29 & 9 & No & 3/month & Right frontal - medial gyrus & Clonic of L arm \\
\hline 6 & 27 & 4 & No & 4/month & $\begin{array}{l}\text { Right frontal (transmantle) - } \\
\text { superior and middle gyrus }\end{array}$ & Hyperkinetic pedal movements \\
\hline 7 & 23 & 4 & No & $10 /$ month & $\begin{array}{l}\text { Right frontal (transmantle) } \\
\text { perirolandic }\end{array}$ & Clonic of L arm \\
\hline 8 & 23 & 4 & Febril seizure & 6/month & $\begin{array}{l}\text { Left fronto parietal } \\
\text { (transmantle) }\end{array}$ & $\begin{array}{l}\text { Somatosensory (tingling) phenomena } \\
\text { of } \mathrm{R} \text { arm }\end{array}$ \\
\hline 9 & 31 & 6 months & Perinatal infection & 2/month & Right temporo-occipital & $\begin{array}{l}\text { mnemonic (deja vu) and dyscognitive } \\
\text { phenomena }\end{array}$ \\
\hline 10 & 33 & 16 & No & 4/month & $\begin{array}{l}\text { Right frontal (transmantle) } \\
\text { middle gyrus }\end{array}$ & Tonic posture of $\mathrm{L}$ arm \\
\hline 11 & 5 & 5 months & Hypoxia & $15 /$ month & Right frontal anterior & Clonic of L arm \\
\hline 12 & 19 & 3 & Hypoxia & 8/month & Left parietal & Tonic posture of $\mathrm{R}$ arm \\
\hline 13 & 19 & 14 & No & 3/month & $\begin{array}{l}\text { Right frontal (transmantle) - } \\
\text { superior gyrus }\end{array}$ & L oculocephalic versive sz \\
\hline 14 & 15 & 9 months & Febril seizure & Seizure free & Right parietal + Right HS & $\begin{array}{l}\text { mnemonic (deja vu) and dyscognitive } \\
\text { phenomena }\end{array}$ \\
\hline 15 & 46 & 17 & No & Seizure free & Right frontal - middle gyrus & L hemiclonic movements \\
\hline 16 & 5 & 5 & No & 3/month & Right frontal - middle gyrus & $\begin{array}{l}\text { Clonic of L arm with jacksonian } \\
\text { march }\end{array}$ \\
\hline 17 & 23 & 3 & No & $14 /$ month & Right frontal anterior & Tonic elevation of $L$ arm \\
\hline 18 & 29 & 1 & Febril seizure & 4/month & Left frontal middle gyrus & Clonic of R arm \\
\hline 19 & 12 & 11 & No & 3/month & Right frontal middle gyrus & Tonic posture of $\mathrm{L}$ arm \\
\hline 20 & 9 & 2 months & No & 3/month & $\begin{array}{l}\text { Right parietal + Left frontal } \\
\text { cavernoma }\end{array}$ & L oculocephalic versive sz \\
\hline
\end{tabular}

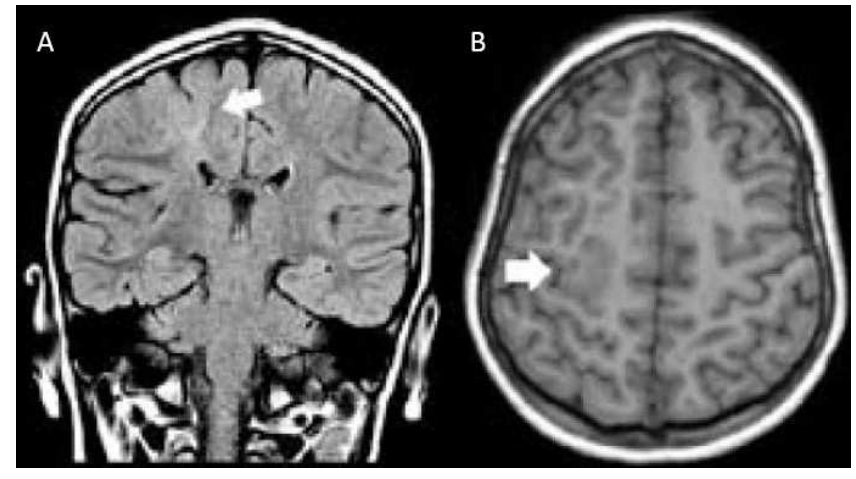

Figure 1. Right precentral focal cortical dysplasia (A) FLAIR coronal view and (B) T1 axial view.

\subsection{MRI}

The frontal lobe was affected in 14 cases (70\%) (Fig. 1). In 10 cases $(50 \%)$ the dysplastic cortex was located on the medial aspect of the brain, and there were no cases affecting both the medial and the lateral surfaces. Three cases involve at least two lobes. Five cases $(25 \%)$ have transmantle characteristics, all of them involving the frontal lobe (Table 1).

\subsection{Response to Treatment and Epilepsy Surgery}

According to the definition proposed by the ILAE [14] all patients, except two subjects had drug resistant epilepsy. The resistant cases failed to achieve sustained seizure freedom with at least two adequate and tolerated AED schedules. With the current scheme of treatment, patients have decreased seizure frequency by more than $50 \%$ and in two patients $(10 \%)$ achieved complete seizure control only with AEDs, one patient in monotherapy with $\mathrm{CBZ}$ and the other with polytherapy with three drugs (VPA, LTG and CNZ). All patients, except two, were on polytherapy, eight $(40 \%)$ having tried 3 or more AEDs in different combinations. The most used AED was CBZ in thirteen patients $(65 \%)$ (Table 2$)$. 
Table 2. AEDs schedules

\begin{tabular}{lll}
\hline Patient & Sz Frequency & AEDs \\
\hline 1 & $5 /$ month & CBZ CNZ \\
2 & $4 /$ month & CBZ TPM \\
3 & $5 /$ month & VPA LTG CNZ \\
4 & $6 /$ month & CBZ TPM FB \\
5 & $3 /$ month & CBZ \\
6 & 4 month & OXC VPA CNZ \\
7 & 10 month & CBZ FB \\
8 & $6 /$ month & CBZ TPM \\
9 & $2 /$ month & VPA TPM CLB \\
10 & $4 /$ month & CBZ LVT \\
11 & $15 /$ month & CBZ TPM \\
12 & $8 /$ month & VPA LTG CNZ \\
13 & $3 /$ month & OXC TPM \\
14 & sz free & VPA LTG CNZ \\
15 & sz free & CBZ \\
16 & $3 /$ month & VPA CNZ \\
17 & 14 month & CBZ TPM \\
18 & $4 /$ month & CBZ CNZ \\
19 & $3 /$ month & CBZ DZP TPM FB DFH \\
20 & $3 /$ month & CBZ LVT CNZ \\
\hline
\end{tabular}

CBZ: carbamacepine, CNZ: clonazepam, FB: phenobarbital, LTG: lamotrigine, LVT: levetiracetam, OXC: oxcarbacepine, TPM: topiramate, VPA: valproic acid, sz: seizure

\section{Conclusions}

Our series of 20 patients with MRI diagnosis of FCD is heterogeneous, with different locations and different response to AEDs.

Almost all patients began their seizure at early childhood. All patients presented focal seizures with clinical semiology concordant with lesional area seen on MRI. Most of them have FCD in the frontal lobe. As mentioned by other authors $[5,6,22,26], \mathrm{FCD}$ is associated with a wide range of clinical presentations. There is no particular seizure semiology that characterizes patients with FCD compared with other surgical series of epilepsy patients [16-18]. There are no distinctive interictal or ictal scalp EEG features that are exclusively associated with FCD. The EEG can show interictal background slowing, interictal spikes and polyspikes, and ictal events with electrographic characteristics similar to other patients with intractable epilepsy who are undergoing presurgical evaluation [19-21].

All patients, except two who became seizures free, have drug-resistant epilepsy according ILAE definition [14]. However, the average low frequency of seizures and no cases of status epilepticus (SE), epilepsia partialis continua (EPC), infantile spasms and reflex seizures, as referred by most series $[16,17]$ reinforces the heterogeneity of these pathology associated to focal epilepsy.

Most published series of patients are surgical or pathological series of pediatric cases [17, 22-26]. Patients have a high frequency of seizure, including several in the day.
Some reports mention EPC [27, 28] or SE [29].

One possible explanation for these findings, including the two free seizure patients, may be a different selection criterion, since most of the published series are surgical $[16,17]$.

This finding emphasizes the heterogeneity of these patients and the importance of the correct use of antiepileptic drugs schemes, as well as it can be dynamic over time.

A proportion of medically resistant patients with cortical dysplasia are poor surgical candidates because the lesion cannot be completely identify or removed if it involves eloquent areas of the cortex. With the development of new drugs and the correct choice of treatment schedules is expected that more patients with cortical dysplasia would be treated successfully without increasing the risk of a surgery.

\section{References}

[1] Marin-Padilla M. Developmental neuropathology and impact of perinatal brain damage. III: gray matter lesions of the neocortex. J Neuropathol Exp Neurol. 1999; 58: 407-29.

[2] Krsek P, Pieper T, Karlmeier A, Hildebrandt M, Kolodziejczyk D, Winkler P. et al. Different presurgical characteristics and seizure outcomes in children with focal cortical dysplasia type I or II. Epilepsia. 2009; 50:125-37.

[3] Chung CK, Lee SK, Kim KJ. Surgical outcome of epilepsy caused by cortical dysplasia. Epilepsia. 2005; 46 Suppl $1: 25-9$.

[4] Semah F, Picot MC, Adam C, Broglin D, Arzimanoglou A, Bazin $\mathrm{B}$, et al. Is the underlying cause of epilepsy a major prognostic factor for recurrence? Neurology. 1998; 51: 1256-62.

[5] Brodtkorb E, Nilsen G, Smevik O, Rinck PA. Epilepsy and anomalies of neuronal migration: MRI and clinical aspects. Acta Neurol Scand. 1992; 86:24-32.

[6] Steffenburg U, Hedstrom A, Lindroth A, Wiklund LM, Hagberg G, Kyllerman M. Intractable epilepsy in a population-based series of mentally retarded children. Epilepsia. 1998; 39:767-75.

[7] Taylor DC, Falconer MA, Bruton CJ, Corsellis JA. Focal dysplasia of the cerebral cortex in epilepsy. J Neurol Neurosurg Psychiatry. 1971; 34:369-87.

[8] Ward LC, Bergman MA, Hebert KR. WAIS-IV subtest covariance structure: conceptual and statistical considerations. Psychol Assess. 2012. 24:328-40.

[9] Berg AT, Berkovic SF, Brodie MJ, Buchhalter J, Cross JH, van Emde Boas W, et al. Revised terminology and concepts for organization of seizures and epilepsies: report of the ILAE Commission on Classification and Terminology, 2005-2009. Epilepsia. 2010; 51:676-85.

[10] Barkovich AJ. Malformations of neocortical development: magnetic resonance imaging correlates. Curr Opin Neurol. 1996; 9:118-21.

[11] Barkovich AJ, Kuzniecky RI. Neuroimaging of focal malformations of cortical development. J Clin Neurophysiol. $1996 ; 13: 481-94$ 
[12] Barkovich AJ, Kuzniecky RI, Dobyns WB. Radiologic classification of malformations of cortical development. Curr Opin Neurol. 2001; 14:145-9.

[13] Blumcke I, Thom M, Aronica E, Armstrong DD, Vinters HV, Palmini A, et al. The clinicopathologic spectrum of focal cortical dysplasias: a consensus classification proposed by an ad hoc Task Force of the ILAE Diagnostic Methods Commission. Epilepsia. 2011; 52:158-74.

[14] Kwan P, Arzimanoglou A, Berg AT, Brodie MJ, Allen Hauser W, Mathern G, et al. Definition of drug resistant epilepsy: consensus proposal by the ad hoc Task Force of the ILAE Commission on Therapeutic Strategies. Epilepsia. 2010; 51: 1069-77.

[15] Glauser T, Ben-Menachem E, Bourgeois B, Cnaan A, Chadwick D, Guerreiro C, et al. ILAE treatment guidelines: evidence-based analysis of antiepileptic drug efficacy and effectiveness as initial monotherapy for epileptic seizures and syndromes. Epilepsia. 2006; 47:1094-120.

[16] Lerner JT, Salamon N, Hauptman JS, Velasco TR, Hemb M, $\mathrm{Wu} \mathrm{JY}$, et al. Assessment and surgical outcomes for mild type I and severe type II cortical dysplasia: a critical review and the UCLA experience. Epilepsia. 2009; 50: 1310-35.

[17] Hauptman JS, Mathern GW. Surgical treatment of epilepsy associated with cortical dysplasia: 2012 update. Epilepsia. 2012; 53 Suppl 4:98-104.

[18] Papayannis CE, Consalvo D, Kauffman MA, Seifer G, Oddo S, D'Alessio L, et al. Malformations of cortical development and epilepsy in adult patients. Seizure. 2012; 21:377-84.

[19] Noachtar S, Bilgin O, Remi J, Chang N, Midi I, Vollmar C, et al. Interictal regional polyspikes in noninvasive EEG suggest cortical dysplasia as etiology of focal epilepsies. Epilepsia. 2008; 49:1011-7.

[20] Seifer G, Blenkmann A, Princich JP, Consalvo D, Papayannis C, Muravchik C, et al. Noninvasive approach to focal cortical dysplasias: clinical, EEG, and neuroimaging features.
Epilepsy Res Treat. 2012;736784.

[21] Blenkmann A, Seifer G, Princich JP, Consalvo D, Kochen S, Muravchik C. Association between equivalent current dipole source localization and focal cortical dysplasia in epilepsy patients. Epilepsy Res. 2012; 98:223-31.

[22] Mathern GW, Giza CC, Yudovin S, Vinters HV, Peacock WJ, Shewmon DA, et al. Postoperative seizure control and antiepileptic drug use in pediatric epilepsy surgery patients: the UCLA experience, 1986-1997. Epilepsia. 1999; 40: 1740-9.

[23] Varotto G, Tassi L, Franceschetti S, Spreafico R, Panzica F. Epileptogenic networks of type II focal cortical dysplasia: a stereo-EEG study. Neuroimage. 2012; 61:591-8.

[24] Sisodiya SM. Surgery for malformations of cortical development causing epilepsy. Brain. 2000; 123:1075-91.

[25] Tassi L, Colombo N, Garbelli R, Francione S, Lo Russo G Mai R, et al. Focal cortical dysplasia: neuropathological subtypes, EEG, neuroimaging and surgical outcome. Brain. 2002; 125:1719-32.

[26] Colombo N, Tassi L, Galli C, Citterio A, Lo Russo G, Scialfa $\mathrm{G}$, et al. Focal cortical dysplasias: MR imaging, histopathologic, and clinical correlations in surgically treated patients with epilepsy. AJNR Am J Neuroradiol. 2003; 24:724-33.

[27] Nakken KO, Server A, Eriksson AS, Kostov H, Lossius R, Haakonsen M. A patient with a 44-year history of epilepsia partialis continua caused by a perirolandic cortical dysplasia. Epilepsy Behav. 2005; 6:94-7.

[28] Nakken KO, Eriksson AS, Kostov H, Lossius R, Haakonsen M, Server A, et al. [Epilepsia partialis continua (Kojevnikov's syndrome)]. Tidsskr Nor Laegeforen. 2005; 125:746-9.

[29] Tan RY, Neligan A, Shorvon SD. The uncommon causes of status epilepticus: a systematic review. Epilepsy Res. 2010; 91:111-22. 